\title{
Le duo entrepreneur investisseur dans les systèmes territoriaux d'innovation. Le rôle de l'agilité.
}

\author{
The Entrepreneur Venture Capitalist Duo in Territorial Innovation \\ Systems. The Role of Agility.
}

\author{
Hélène Perrin Boulonne ${ }^{1}$ \\ ${ }^{1}$ ULCO, Clersé UMR 8019, helenepboulonne@gmail.com
}

\begin{abstract}
RÉSUMÉ. L'investisseur en capital est le principal investisseur de l'entreprise innovante. La mise en relation de ces deux acteurs est favorisée par les systèmes territoriaux d'innovation. L'investisseur joue un rôle important à la fois comme apporteur de fonds et comme accompagnateur de l'entrepreneur. L'article propose une nouvelle définition de la relation entrepreneur investisseur, le duo entrepreneur investisseur, qui apporte une vision complémentaire à la théorie de l'agence habituellement utilisée pour décrire cette relation. L'article s'intéresse également au rôle de l'agilité dans le fonctionnement de ce duo et montre l'importance de sa contribution au capital savoir territorial par l'agrégation des connaissances et des compétences de l'entrepreneur et de l'investisseur.

ABSTRACT. The venture capitalist is the main funder of the innovative firm. The relationship between these two actors is favored by territorial innovation systems. The venture capitalist plays an important role both as a provider of funds and as an advisor of the entrepreneur. This article proposes a new definition of the entrepreneur venture capitalist relationship, the "entrepreneur venture capitalist duo", which brings a complementary vision to the agency theory, usually used to describe this relationship. The article also looks at the role of agility in the functioning of this duo, and shows the importance of its contribution to the territorial knowledge capital through the accumulation of knowledge and skills of the entrepreneur and the venture capitalist.

MOTS-CLÉS. Entrepreneur, capital-risque, agilité, duo entrepreneur investisseur, capital savoir territorial.

KEYWORDS. Entrepreneur, venture capital, agility, entrepreneur venture capitalist duo, territorial knowledge capital.
\end{abstract}

\section{Introduction}

Cet article propose d'analyser dans quelle mesure les caractéristiques de l'agilité (individus et interactions, collaboration et adaptation au changement) peuvent s'appliquer aux relations entrepreneur investisseur dans les systèmes territoriaux d'innovation. Le sytème territorial d'innovation est défini comme : l'ensemble des acteurs, publics et privés, interagissant sur un territoire donné pour favoriser le développement de l'innovation et des entreprises situées sur ce même territoire [COO 92]. L'investisseur est pris au sens de l'investisseur en capital qui est un acteur clé du développement des économies avancées. Le capital-risque soutient l'économie basée sur la connaissance et l'innovation en permettant la création et le développement d'entreprises [GOM 01] ; [MAS 11] ; [FLO 88]. Le capitalrisque a contribué au développement de clusters comme la Silicon Valley ou la route 128 [FLO 88]; [SAX 94]. Dans ce cadre, deux hypothèses peuvent être posées : i) Les relations entre entrepreneurs et investisseurs sont multiples et s'inscrivent dans des logiques d'interactions, d'apprentissages et de collaborations plus que dans des logiques d'asymétries d'informations au regard de la théorie de l'Agence, ce qui permet plus d'agilité, ii) les relations s'inscrivent dans une dialectique avec le territoire.

La première partie définit le capital-risque, financeur de la création d'entreprise innovante au sein des systèmes territoriaux d'innovation. Le financement en capital-risque s'articule autour de deux acteurs, l'entrepreneur et l'investisseur. Leur relation est présentée sous l'angle de la théorie de l'agence, s'appuyant sur les asymétries d'informations existantes entre entrepreneur et investisseur. Cette relation est ensuite appréhendée en dépassant la relation contractuelle, le rôle de l'investisseur allant au-delà du simple financement. 
Dans une deuxième parie nous proposons une nouvelle définition, plus « agile », de la relation entrepreneur investisseur, appelée le " duo entrepreneur investisseur ». Le " duo entrepreneur investisseur » se définit selon nous comme "l'ensemble des interactions, la mise en relation et l'apprentissage mutuel entre investisseurs et entrepreneurs ». Le duo entrepreneur investisseur est rapproché de la théorie de l'entrepreneuriat par débordement de connaissances (knowledge spillover entrepreneurship, KSE) qui considère l'entrepreneur comme un transformateur de connaissance en entreprises [AUD 11]. Le rôle de l'agilité dans le fonctionnement du duo est ensuite analysé. Enfin, nous présentons l'apport du « duo entrepreneur investisseur » au « capital savoir territorial » définit comme «l'ensemble des informations et connaissances scientifiques et techniques produites, acquises, combinées et systématisées par les entreprises et les institutions locales pour être utilisées dans un processus de création de valeur et selon un projet territorial défini » [LAP 17].

\section{L'investisseur en capital et l'entrepreneur dans les systèmes territoriaux d'innovation : des relations souples, mais soutenues}

\subsection{L'investisseur en capital est le principal investisseur de l'entreprise innovante}

La question du financement de l'entreprise innovante est abordée dans les travaux de Joseph Schumpeter comme une condition nécessaire au processus d'innovation. Le financement permet une réallocation des ressources nécessaires à la réalisation de changements radicaux dans la structure des activités économiques. Schumpeter [SCH 34] décrit le banquier comme essentiel au processus de développement, il se place entre ceux qui souhaitent former de nouvelles combinaisons et ceux qui détiennent les moyens de production, et rend possible au nom de la société ces nouvelles combinaisons, il est qualifié de « ephor of the exchange economy » ( [SCH 34] p.74).

Les investisseurs habituels de l'entreprise, les banques, l'autofinancement, le financement par les proches sont peu ou pas présents sur les secteurs de l'innovation et notamment au démarrage de l'entreprise et dans les premières phases de développement des produits et services [GOM 99]. Ce financement est donc pour l'essentiel assuré par des investissements privés (personnes physiques ou morales) et publics au capital de l'entreprise [GOM 99]. Ce type de financement est couramment appelé capital-risque ou financement en capital. Le capital-risque peut prendre différentes formes selon l'origine des fonds et selon les acteurs en charge d'investir les fonds qui ne sont pas nécessairement une seule et même entité. Il consiste à prendre des participations majoritaires ou minoritaires dans le capital de petites et moyennes entreprises non cotées. Le capital-risque intervient : au moment de la création d'entreprises grâce au capital amorçage, dans le cadre du développement de l'entreprise par le financement de l'innovation grâce au capital-innovation.

Il est largement admis dans la littérature que le financement en capital est un élément clé du développement des économies avancées. En effet, le financement en capital soutient l'économie basée sur la connaissance et l'innovation en permettant la création et le développement d'entreprises. Il a été montré de façon empirique que le financement en capital a contribué à $10 \%$ des innovations technologiques dans la décennie 1983-1992 aux États-Unis, alors qu'il ne représentait que 3\% des montants investis en R\&D [GOM 01], [MAS 02], [KOR 00]. De nombreux travaux ont montré l'importance du capital-risque dans le développement d'entreprises innovantes dans des territoires innovants [FLO 88], [SAX 94]. La plupart de ces travaux se trouvent dans les théories des clusters et des écosystèmes d'innovation. Dans des lieux tels que les clusters, la présence du financement en capital est considérée comme un catalyseur pour l'entrepreneuriat local, et comme prenant part au développement des systèmes territoriaux d'innovation. Le cluster peut être défini comme du capital humain et intellectuel façonné par le capital social et soutenu par le capital financier [CAR 09]. Le capital-risque a contribué au développement de clusters comme la Silicon Valley ou la route 128, [SAX 94]. Le capital-risque peut être considéré comme un élément favorisant la création et le développement d'entreprises innovantes dans les systèmes territoriaux d'innovation, [PER 17]. Le concept de système territorial d'innovation est défini comme l'ensemble des acteurs, publics et privés, 
interagissant sur un territoire donné pour favoriser le développement de l'innovation et des entreprises situées sur ce même territoire [COO 01]. Quatre raisons justifient le fait que le capital-risque est le plus approprié pour le financement des entreprises à haut potentiel : le capital-risque est le mieux placé pour détecter les entreprises qui vont réussir, le capital-risque apporte de la valeur ajoutée par son activité de monitoring, le soutien par le capital-risque donne un signal positif aux autres parties prenantes et le capital-risque apporte son réseau à l'entrepreneur [GRI 14].

Le financement en capital-risque de la création d'entreprise s'articule autour de deux acteurs, l'entrepreneur et le investisseur. Les relations entre ces deux acteurs sont complexes.

\subsection{La relation entrepreneur investisseur sous l'angle de théorie de l'agence}

Dans le domaine des sciences économique, la relation entrepreneur investisseur est le plus souvent appréhendée au regard de la théorie de l'agence. Une relation d'agence se définit comme un contrat par lequel une personne (le principal) engage une autre personne (l'agent) pour exécuter en son nom une tâche quelconque qui implique une délégation d'un certain pouvoir de décision à l'agent [JEN 76]. Cette relation recouvre en fait toute relation entre deux individus telle que la situation de l'un dépende d'une action de l'autre : l'individu qui agit est l'agent, la partie affectée est le principal. Les problèmes qu'étudie la théorie de l'agence n'apparaissent cependant que dans la mesure où les intérêts des deux parties peuvent diverger, et surtout où il $\mathrm{y}$ a information imparfaite et asymétrie d'information entre les acteurs. Les problèmes peuvent se classer en trois catégories : l'anti-sélection (le principal ignore une information sur l'agent qui a un impact sur le contrat), le hasard moral (l'agent s'engage à accomplir une action qui influe sur le résultat final, l'action dépend d'un paramètre que connaît uniquement de l'agent), et le signal (l'agent veut donner une information au principal qui n'est pas vérifiable) [AKE 70].

Le modèle peut s'appliquer au cas du financement de l'entreprise innovante. Les travaux cités dans la suite de l'article font tous l'hypothèse que le principal est l'investisseur et l'entrepreneur l'agent. En effet, le financement des entreprises innovantes est particulièrement complexe en raison des imperfections de marchés qui sont le fait de fortes asymétries d'informations entre entrepreneurs et investisseurs. L'une des parties dispose d'informations sur la transaction qui ne sont pas connues des autres parties prenantes, généralement il s'agit de l'entrepreneur qui connaît les capacités de développement de son entreprise et les présente dans son business plan. Le caractère technologique renforce cet effet, plus la technologie est complexe plus les coûts d'analyses (due diligence) et les risques pour les investisseurs sont élevés.

Ceci conduit à des risques d'anti-sélection de la part des investisseurs. Akerlof, [AKE 70], en prenant exemple du marché des voitures d'occasion, analyse le manque d'information d'une des parties d'un contrat qui conduit à n'avoir sur le marché que des voitures avec des défauts cachés. Il y a un risque pour L'investisseurde mal évaluer le potentiel et les fonds nécessaires au projet d'entreprise. L'investisseur qui ne détient pas suffisamment d'information sur l'entreprise, aura tendance à n'investir que sur les projets les moins risqués mais également les moins prometteurs, les meilleurs projets d'entreprises sortiront du marché. C'est ainsi que certains secteurs sortent du marché du financement en capital, c'est le cas des technologies vertes [NAN 15].

On observe également des problèmes de hasard moral [BER 98]. L'investisseurn'a pas la capacité de contrôler si l'entrepreneur met en œuvre de façon efficace tous les moyens pour la réussite de l'entreprise. Dans un cas extrême l'entrepreneur peut profiter du système pour lever des fonds alors qu'il sait qu'il va faire faillite. Ce problème de passager clandestin est mis en évidence dans certains travaux [ELI 09]. Les problèmes de hasard moral conduisent à la rédaction de contrats complexes et donc coûteux, augmentant ainsi les coûts de transaction. 
Enfin, les problèmes de signaux interviennent lorsque l'entrepreneur veut montrer ses capacités de réussite au investisseur, sans avoir les moyens de le prouver. Ce problème peut être résolu par la labélisation des projets de création d'entreprises.

Sur la base de cette théorie l'analyse de la relation entrepreneur investisseur se réduit le plus souvent à l'étude du business plan produit par l'entrepreneur, et à l'étude de la capacité d'analyse du business plan par l'investisseur (due diligence). L'investisseur ne dispose jamais de toute l'information. De ces problèmes d'asymétries d'informations découlent des coûts de transaction élevés : chaque transaction entre entrepreneur et investisseur se heurte à l'opportunisme de chacun, l'entrepreneur et Les investisseurs recherchent leur intérêt personnel [CAB 97]. L'entrepreneur et l'investisseur ne partagent pas la même vision du risque : pour l'entrepreneur généralement actionnaire, il risque de tout perdre alors que l'investisseur dilue son risque au milieu d'autres opérations de financement. De même les motivations de l'un et l'autre pour s'engager dans le projet sont différentes ; l'incitation pour l'investisseur est l'espoir d'un gain financier au moment de la sortie, pour l'entrepreneur c'est la possibilité de disposer d'un accès au capital malgré un niveau de risque élevé.

Les travaux analysant les problématiques d'asymétries d'informations entre entrepreneur et investisseur, montrent l'importance d'une coopération sur le long terme entre les deux agents. Sur la base du dilemme du prisonnier, il a été montré que l'investisseur et l'entrepreneur ont une incitation à collaborer pour arriver à une situation jugée optimale [CAB 97]. Une relation de long terme, incluant un apprentissage mutuel, des conseils en matière de management, y compris le remplacement de l'équipe managériale diminue les risques de hasard moral et améliorent ainsi les chances d'un bon déroulement de la relation contractuelle entre les deux parties [HEG 98]. La relation de long terme, en permettant une meilleure connaissance mutuelle, diminue l'asymétrie d'information et facilite l'agilité, en effet une meilleure connaissance de l'entrepreneur par l'investisseur, lui permet de s'adapter plus facilement à ses besoins.

Il est donc important d'étudier de quelles façons entrepreneur et investisseur peuvent collaborer dans l'objectif de faire réussir le projet d'entreprise.

\subsection{L'investisseur en capital : un rôle au-delà du financement qui rend la relation entrepreneur investisseur effective}

Le capital-risque comporte dans son rôle vis-à-vis de l'entrepreneur une composante financière et une composante non financière. La partie non financière de l'investissement est essentielle. La composante non financière de l'intervention des investisseurs joue un rôle dans la gestion de l'entreprise et joue également un rôle en envoyant un signal positif aux parties prenantes de l'entreprise innovante [FLO 88]. En effet, le financement en capital est différent du financement par emprunt bancaire, l'emprunt bancaire est encadré juridiquement et soumis à un plan de remboursement précis qui n'est pas lié à la réussite de l'entreprise. L'emprunt est généralement garanti par le capital de l'entreprise et très souvent par les biens propres de l'entrepreneur. Dans le cas du financement en capital dans l'entreprise, le remboursement et le gain pour l'investisseur sont liés à la croissance et au succès de l'entreprise. L'investisseur devient dès lors un partenaire incontournable de l'entrepreneur, associé à la réussite de l'entreprise. Le rôle de partenaire se traduit de plusieurs manières et trouve ses limites dans les asymétries d'informations qui existent entre investisseurs et entrepreneurs. L'investisseur agit d'une part en facilitant l'accès aux réseaux et aux marchés et d'autre part en apportant une valeur ajoutée en matière de gestion de l'entreprise [MCM 87].

Les investisseurs en capital lorsqu'ils s'inscrivent dans une proximité géographique avec les entrepreneurs peuvent réduire les asymétries d'informations par une connaissance locale des marchés [KLA 05]. Le capital-risque peut faciliter l'entrepreneuriat innovant, dans la mesure où un réseau d'investisseurs en capital développé apporte une incitation essentielle pour les entrepreneurs en réduisant les difficultés pour entrer sur le marché. Les investisseurs utilisent expériences et contacts et réduisent les coûts d'information et d'opportunités [FLO 88]. 
L'expertise en matière de gestion permet de réduire les problèmes de hasard moral dès lors que L'investisseur est partie prenante de la réussite du projet. Les porteurs de projets d'entreprises innovantes notamment lorsqu'ils sont issus de la recherche, ne sont pas nécessairement des gestionnaires. Les investisseurs en capital locaux facilitent la création de start-ups universitaires en investissant des capitaux et en les aidant à trouver les personnes ayant l'expertise pour gérer les entreprises [MCD 05]. L'intervention de société de capital-risque ou de business angels dans une startup issue de l'université augmente ses chances de succès [SHA 02]. De nombreuses études empiriques dont nous donnons quelques exemples dans la suite du paragraphe, montrent les effets bénéfiques sur le développement de l'entreprise, qui se traduisent par : des délais de mise sur me marché plus courts, une croissance de l'emploi et des revenus plus rapides, une capacité à exporter plus importante et un nombre de dépôts de brevets plus élevés. Sur 170 entreprises de la Silicon Valley qui poursuivent une stratégie d'innovation, il a été montré que les entreprises financées par le capital-risque ont des délais de mise sur le marché de leur innovation plus courts [HEL 00]. L'étude de plusieurs pays montrent clairement une relation positive entre le financement par le capital-risque et la croissance de l'entreprise [MAN 13]. Des études comparant des entreprises financées et non financées par le capitalrisque montrent que celles financées ont des revenus qui croissent plus rapidement [PUR 12]. Les entreprises financées ont également une croissance de leurs valeurs et emplois plus importantes [CHE 11]. Les entreprises financées ont une plus grande propension à exporter [HEL 02]. Elles ont également tendance à déposer plus de brevets, aux États-Unis, un dollar investi par le capital-risque apparaît être 3 à 4 fois plus efficace pour stimuler le dépôt de brevet qu'un dollar investi par les grandes entreprises dans la R\&D [KOR 00].

L'investisseur en capital joue donc un rôle important, à la fois en tant qu'apporteur de capitaux et en tant qu'accompagnateur de l'entrepreneur. La relation entre l'entrepreneur et son investisseur va ainsi être un élément important dans le succès du projet d'entreprise. Par rapport à la relation avec le banquier traditionnel, la relation présente un caractère agile, l'aspect non financier jouant un rôle prépondérant [FLO 88]. Dans la deuxième partie, nous nous intéressons à cette relation que nous nommons le duo entrepreneur investisseur.

\section{Une conception fondée sur l'agilité du duo entrepreneur investisseur dans les Systèmes territoriaux d'innovation}

\subsection{Le duo entrepreneur investisseur et sa contribution à l'économie du territoire}

Il s'agit donc de proposer une vision complémentaire à la théorie de l'agence des relations entrepreneurs et investisseurs en proposant une approche faisant appel à la collaboration et à une contractualisation adaptable dans le cadre d'un rapport de confiance. Le contrat entre L'investisseur et l'entrepreneur rendu nécessaire par le mode de financement en capital doit permettre un mode de fonctionnement sur la période de financement souple et adaptable à l'incertitude inhérente à la création d'entreprise innovante. On dépasse alors une vision où tout se joue avant la contractualisation avec d'un côté la due diligence mise en place par l'investisseur et de l'autre le business plan proposé par l'entrepreneur. L'entrepreneur et l'investisseur doivent prendre des décisions basées sur des évaluations d'un futur possible. Le rôle de l'investisseur peut s'analyser au-delà de sa capacité à choisir les projets entrepreneuriaux les plus viables si l'on se projette dans un temps plus long, c'est à dire sur la durée du financement de 5 à 7 ans.

La problématique de la décision d'investissement et du choix de l'investisseur n'est plus appréhendée uniquement à l'instant $t$ comme une décision d'investissement mais comme un processus sur plusieurs années allant jusqu'à la sortie de l'investisseur. La période post-investissement est essentielle. Dès lors il s'agit de comprendre les processus qui permettent de promouvoir la coopération entre l'entrepreneur et l'investisseur sur l'ensemble de la période. 
Nous proposons de nommer cette relation sur l'ensemble de la période de financement le duo entrepreneur investisseur que nous définissons comme " la mutualisation des ressources et compétences dans une relation de confiance et de coopération dans la poursuite d'un objectif commun qui est la réussite de l'entreprise. Il est constitué de l'ensemble des interactions, de la mise en relation et de l'apprentissage mutuel entre investisseurs et entrepreneurs ». L'analyse des interactions et des apprentissages entre entrepreneur et investisseur se positionne dans le cadre de la géographie économique évolutionniste [BOS 04] qui cherche à comprendre les actions des acteurs économiques et les sentiers d'évolution dans un contexte temporel et spatial. Des travaux allant dans le sens de cette définition sont menés dans le cadre de l'analyse cognitive de l'entrepreneuriat. Il est proposé de dépasser les problématiques de la théorie de l'agence, et d'analyser les relations entrepreneurs investisseurs en intégrant les concepts de " coûts et valeurs cognitifs » [WIR 10]. Les coûts cognitifs sont liés à l'absence de compréhension de la part du investisseur du schéma de pensée de l'entrepreneur et de sa façon de percevoir les opportunités, la valeur cognitive peut être obtenue de la part des investisseurs expérimentés. Il conclut à l'intérêt pour l'entrepreneur de favoriser des situations où il a un lien fort avec son investisseur ( «investors relationship ») au détriment des investisseurs non impliqués («arm's lenght financing »).

L'entrepreneur peut être défini par une " équation entrepreneuriale »: incertitude + risque + innovation [BOU 17]. L'incertitude dans laquelle évolue l'entrepreneur est atténuée par les connaissances du investisseur (marchés et concurrence), la prise de risque de l'entrepreneur peut être réduite par la vision stratégique et les conseils financiers du investisseur. A l'inverse les compétences de l'entrepreneur peuvent pousser L'investisseur à prendre plus de risque et à financer des projets à plus haut potentiels, et s'engager sur de nouvelles technologies. L'utilisation des innovations issues de la production de connaissance par l'entrepreneur est favorisée par la mise à disposition par l'investisseur de compétences en matière de conseils et de ressources humaines. L'accès à des réseaux d'entrepreneurs pour l'investisseur peut être source d'opportunité. La mise en commun de ces compétences pour former le duo entrepreneur investisseur est favorisée par l'appartenance à un système territorial d'innovation. En retour le duo entrepreneur investisseur va contribuer au fonctionnement du système territorial d'innovation. La figure 1 ci-dessous montre la complémentarité des compétences de l'investisseur et de l'entrepreneur et le résultat de leur mise en commun au sein du système territorial d'innovation. 


\begin{tabular}{|l|l|l|}
\hline FINANCEUR & DUO ENTREPRENEUR-FINANCEUR & ENTREPRENEUR \\
\hline $\begin{array}{l}\text { Vision stratégique } \\
\text { Conseils financiers } \\
\text { Informations sur les marchés } \\
\text { Informations sur la concurrence } \\
\begin{array}{l}\text { Connaissance en ressources humaines } \\
\text { Mise en relation avec des conseils } \\
\text { Mise en relation avec des autres } \\
\text { entreprises }\end{array}\end{array}$ & $\begin{array}{l}\text { Confiance } \\
\text { Proximité } \\
\text { Intérêt collectif pour le } \\
\text { développement économique local } \\
\text { Apprentissage } \\
\text { Echange d'information }\end{array}$ & $\begin{array}{l}\text { Capacité à accepter l'incertitude } \\
\text { Prise de risque } \\
\text { Capacité d'innovation } \\
\text { Connaissances des nouvelles } \\
\text { technologies } \\
\text { Evaluer et exploiter de nouvelles } \\
\text { opportunités } \\
\text { Combinaison de ressources } \\
\text { Appartenances à des réseaux } \\
\text { d'entrepreneurs }\end{array}$ \\
\hline
\end{tabular}

\begin{tabular}{|l|}
\hline Réduction des asymétries \\
d'informations \\
Réduction de l'incertitude \\
Accroissement des capacités \\
relationnels \\
Augmentation du potentiel de \\
financeurs \\
Augmentation du potentiel \\
d'entreprises financées. \\
Création de nouvelles institutions \\
locales
\end{tabular}

Figure 1. Les compétences de l'investisseur, de l'entrepreneur et la mise en commun des compétences au travers du duo entrepreneur investisseur dans un système territorial d'innovation. Source : Auteur.

La confiance, la proximité et l'intérêt commun pour le développement économique local vont permettre de réduire les incertitudes et les asymétries d'informations, et d'augmenter les capacités relationnelles. Par l'apprentissage mutuel, l'entrepreneur peut devenir investisseur et vis-versa. La mise en relation avec des réseaux d'entrepreneurs peut permettre aux investisseurs de mieux appréhender de nouveaux domaines technologiques. Les échanges d'informations, de réseaux peuvent permettre de créer de nouvelles institutions locales telles que des réseaux de business angels.

Le rôle essentiel de l'investisseur dans le processus de création d'entreprise innovante présenté dans la première partie nous conduit à poser l'hypothèse selon laquelle c'est le duo entrepreneur investisseur qui permet d'exploiter les débordements de connaissance en facilitant la transformation de la connaissance en nouvelles entreprises et donc en croissance.

Le débordement de connaissance est défini comme une externalité, avec des agents qui produisent des connaissances dont ils ne sont pas capables de tirer tous les bénéfices. On peut retenir la définition de Grossman et Helpman (1993 pp.16) [GRO 93]: «By technological spillovers, we mean that firms can acquire information created by others without paying for that information in a market transaction, and the creators (or current owners) of the information have no effective recourse, under prevailing laws, if other firms utilize information so acquired ». Le débordement de connaissance augmente le champ des opportunités pour les entreprises existantes. Le débordement de connaissance est une caractéristique des clusters et des systèmes territoriaux d'innovation.

Au travers du « paradoxe européen », les tenants de la théorie du « knowledge spillover entrepreneurship » (KSE) montrent que la connaissance ne joue pas nécessairement son rôle dans la croissance endogène [ACS 09]. En effet une croissance modeste malgré des niveaux élevés d'investissement en 
capital humain et en recherche et développement caractérise beaucoup de pays européen. Ce constat va à l'encontre des résultats attendus selon la théorie de la croissance endogène. Les tenants de la KSE l'expliquent par la faible dynamique entrepreneuriale en Europe. La KSE applique la notion de débordement de connaissance à l'entrepreneur qui oriente la connaissance et lui permet de jouer son rôle pour favoriser la croissance. Les nouvelles entreprises facilitent l'effet de spillover (débordement) de la connaissance et contribue à la croissance [ACS 09]. L'entrepreneuriat est un des mécanismes qui convertit le savoir en croissance [ACS 12]. C'est en commercialisant des idées qui n'auraient pas été utilisées et en les introduisant sur le marché que les entrepreneurs génèrent de la croissance [ACS 09].

La contribution positive du duo entrepreneur investisseur à l'économie est également corroborée par les travaux qui ont démontré le rôle important du financement pour augmenter la $R \& D$, et par conséquent l'impact positif de la finance entrepreneuriale sur la croissance économique [KIN 93]. Les tenants d'un lien entre innovation et financement, considèrent que les investisseurs renforcent l'innovation en fournissant d'une part des services financiers et d'autre part des informations et une gestion du risque qui diminuent les coûts de transactions et ainsi rendent plus facile les investissements risqués dans les activités entrepreneuriales innovantes [LEV 97]. L'effet du développement financier sur les innovations dans 51 pays entre 1993 et 2008 a été étudié [MEI 14], en cohérence avec les attentes du modèle schumpétérien, en matière de financement, d'entrepreneuriat et de croissance économique, il montre qu'un niveau élevé de développement financier coïncide avec une activité d'innovation plus forte. Il montre également que le système financier est une part importante des infrastructures de l'innovation d'un pays.

\subsection{Le duo entrepreneur investisseur : une conception agile de la relation entrepreneur investisseur.}

L'agilité est définie par trois caractéristiques fondamentales, individus et interactions plutôt que processus et outils, collaboration plutôt que négociation contractuelle, et adaptation au changement. L'agilité a été définie dans le cadre de l'entreprise comme une nouvelle forme d'organisation pour faire face à un environnement de plus en plus instable, marqué par une libéralisation des économies, une augmentation des échanges commerciaux et une intensification de la concurrence [BAR 11].

L'essentiel de la littérature qui fait référence à l'agilité en sciences sociales se concentre sur les questions d'organisations des entreprises. Les entreprises agiles sont définies comme étant capables de réagir rapidement et de façon efficace à toutes sortes de contraintes afin d'assurer une croissance durable des bénéfices et une meilleure insertion dans l'environnement. Dans les conditions rendant l'entreprise agile on trouve l'importance des systèmes d'informations [COR 03], et la constitution de partenariats stratégiques et de réseaux d'apprentissage en vue de développer des capacités dynamiques [BES 04]. L'agilité peut être vue comme une forme d'acceptation de l'aléatoire et une capacité à apprendre de ses erreurs pour progresser sans cesse. On trouve cette vision de l'agilité dans la littérature sur la gestion de crise. On peut rapprocher cette situation de la forte incertitude qui réside dans les choix d'investissement pour les investisseurs en capital-risque et dans les aléas et crises qui surviennent durant toute la période où l'investisseur est présent au capital de l'entreprise.

Le concept de l'entreprise agile est défini comme un processus conduisant à un idéal organisationnel : celui de l'entreprise agile qui se caractérise par la coordination horizontale, le partage de l'information et une grande flexibilité à court terme [BAD 97]. L'agilité est la capacité d'adaptation permanente de l'entreprise en réponse à un environnement caractérisé par la complexité, la turbulence et l'incertitude [GOL 95]. Elle donne les moyens de réagir rapidement au changement, mais également d'agir et de maitriser ce dernier, grâce à d'importantes capacités d'anticipation, d'innovation et d'apprentissage.

Le concept d'agilité peut être rapproché de notion de capacités dynamiques. Proposé par Teece, Pisano et Shen [TEE 97], le concept de capacités dynamiques désigne la capacité à intégrer, construire et reconfigurer des ressources et compétences dans un environnement turbulent. Nous pouvons considérer que la capacité à échanger/partager des compétences entre entrepreneur et investisseur est (c) 2018 ISTE OpenScience - Published by ISTE Ltd. London, UK - openscience.fr 
une capacité dynamique. En effet les échanges entre entrepreneur et investisseur sont de facto dynamiques puisqu'elles visent à mettre en commun des compétences visant à répondre aux évolutions du marché et aux évolutions de l'entreprise financée.

Ainsi, la vision des relations entre entrepreneur et investisseur sous l'angle d'un duo agile plutôt que sous une forme contractuelle doit permettre une meilleure coordination, un partage d'informations et une forte adaptabilité pour faire face aux aléas économiques, sociaux et environnementaux auxquels la nouvelle entreprise doit faire face.

Dans certains cas, seul des processus d'apprentissage mutuel entre l'investisseuret l'entrepreneur peuvent permettre le développement de nouveaux secteurs pour s'adapter à de nouveaux enjeux. C'est le cas en particulier dans le secteur des technologies vertes, les investisseurs doivent investir dans des secteurs sur lesquels ils n'ont aucun savoir leur permettant d'évaluer les risques. Nanda, Younge et Fleming [NAN 15] montrent que dans le secteur des technologies vertes le niveau d'incertitude est trop élevé pour les investisseurs. L'entrepreneur dans un processus de partage des connaissances peut leur permettre d'acquérir les compétences qui leur permettront d'investir dans d'autres projets d'entreprises en améliorant leur connaissance de nouveaux secteurs.

Si l'innovation agile se définit comme un processus souple transformant les individus, leur organisation et leur environnement, le duo entrepreneur investisseur peut prendre part à ce processus. Le duo est caractérisé par sa capacité d'adaptation aux évolutions sociales, économiques et environnementales. Il se caractérise par sa capacité à appréhender l'imprévisible grâce à l'agrégation de compétences. Ces caractéristiques du duo entrepreneur investisseur lui permettent de jouer un rôle dans le territoire.

\subsection{La contribution du duo entrepreneur investisseur au capital savoir territorial}

Le GREMI (Groupe de Recherche sur les Milieux Innovateurs) [AYD 85] met l'accent sur le rôle des interactions dans les processus d'innovation. Les interactions sont également présentes dans les systèmes territoriaux d'innovation selon la définition de Cooke [COO 01]. Pour tirer parti de son appartenance à un système territorial d'innovation, l'entreprise doit être capable d'absorber des nouvelles compétences et connaissances qui se trouvent dans son environnement, lui permettant ainsi d'enrichir son capital savoir. Le " capital savoir » de l'entreprise correspond à « l'ensemble des informations et connaissances scientifiques et techniques produites, acquises, combinées et systématisées pour être utilisée dans un processus de production de valeur [LAP 07], [LAP 16].

Le concept de capital savoir peut s'appliquer au territoire. On utilisera alors le concept de « capital savoir territorial » définit comme : «l'ensemble des informations et connaissances scientifiques et techniques produites, acquises, combinées et systématisées par les entreprises et les institutions locales pour être utilisées dans un processus de création de valeur, et selon un projet territorial défini » [LAP 17]. Le capital savoir territorial détermine le potentiel d'innovation du territoire dans le sens où les acteurs (entrepreneurs, grandes entreprises, institutions locales, investisseurs) puisent dans cette base de connaissances pour créer, développer et diffuser de nouveaux produits et services.

Le duo entrepreneur investisseur joue un rôle clé dans la construction, l'enrichissement et la valorisation du capital savoir territorial : l'entrepreneur associé à l'investisseur transfère le savoir (d'entreprises anciennes ou de plus grandes tailles vers des entreprises nouvelles, de la recherche vers le marché), produit des connaissances nouvelles et recombine les connaissances existantes par l'agrégation de leurs compétences. La production de connaissance est une condition nécessaire à l'existence d'un système territorial d'innovation. Pour que l'innovation induise de l'activité économique, elle doit se traduire par des créations d'entreprises ou de la création d'emplois dans les entreprises existantes par le biais de la commercialisation de nouveaux biens et services. Le développement de nouveaux biens et services, nécessite le plus souvent une prise de risque financière pour l'entrepreneur, ce qui rend la présence du duo entrepreneur investisseur nécessaire au fonctionnement du système territorial d'innovation. 
Dans l'approche évolutionniste [BOS 04], la dynamique du territoire est le fruit d'une évolution conjointe des entreprises et institutions qui interagissent localement. Le duo entrepreneur investisseur apparaît comme un élément clé de cette dynamique.

Les travaux d'E. Dubocage [DUB 06] montrent l'importance des processus d'apprentissage pour l'investisseur: "Que ce soit pour sélectionner les start-ups de bonne qualité ou pour les valoriser de façon pertinente, le capital-risqueur doit être un expert. Les diplômes requis sont d'une double nature : financière et technologique. Mais, c'est essentiellement la pratique qui fera de lui un professionnel avisé, compétent. L'expérience joue donc un rôle décisif dans la mesure où les compétences s'acquièrent par l'apprentissage » ([DUB 06], pp 16). Ces processus d'apprentissage sont favorisés par l'appartenance à des systèmes territoriaux d'innovation [CAR 14].

Le schéma ci-dessous (figure 2) résume les processus en œuvre et les acteurs présents au sein du système territorial d'innovation, et intègre le duo entrepreneur investisseur comme un acteur à part entière du processus. Le système territorial est constitué d'un ensemble de mécanismes (réseaux, apprentissage et interactions) et d'acteurs (producteurs de connaissance, entreprises, institutions locales et investisseurs). Les processus en œuvre au sein du système territorial d'innovation, constitue le " capital savoir territorial ». L'ensemble des acteurs, universités, institutions locales, entreprises, le duo entrepreneur investisseur, contribue à enrichir le capital savoir territorial en produisant, utilisant et recombinant la connaissance. Ce processus donne lieu à la production d'innovation.

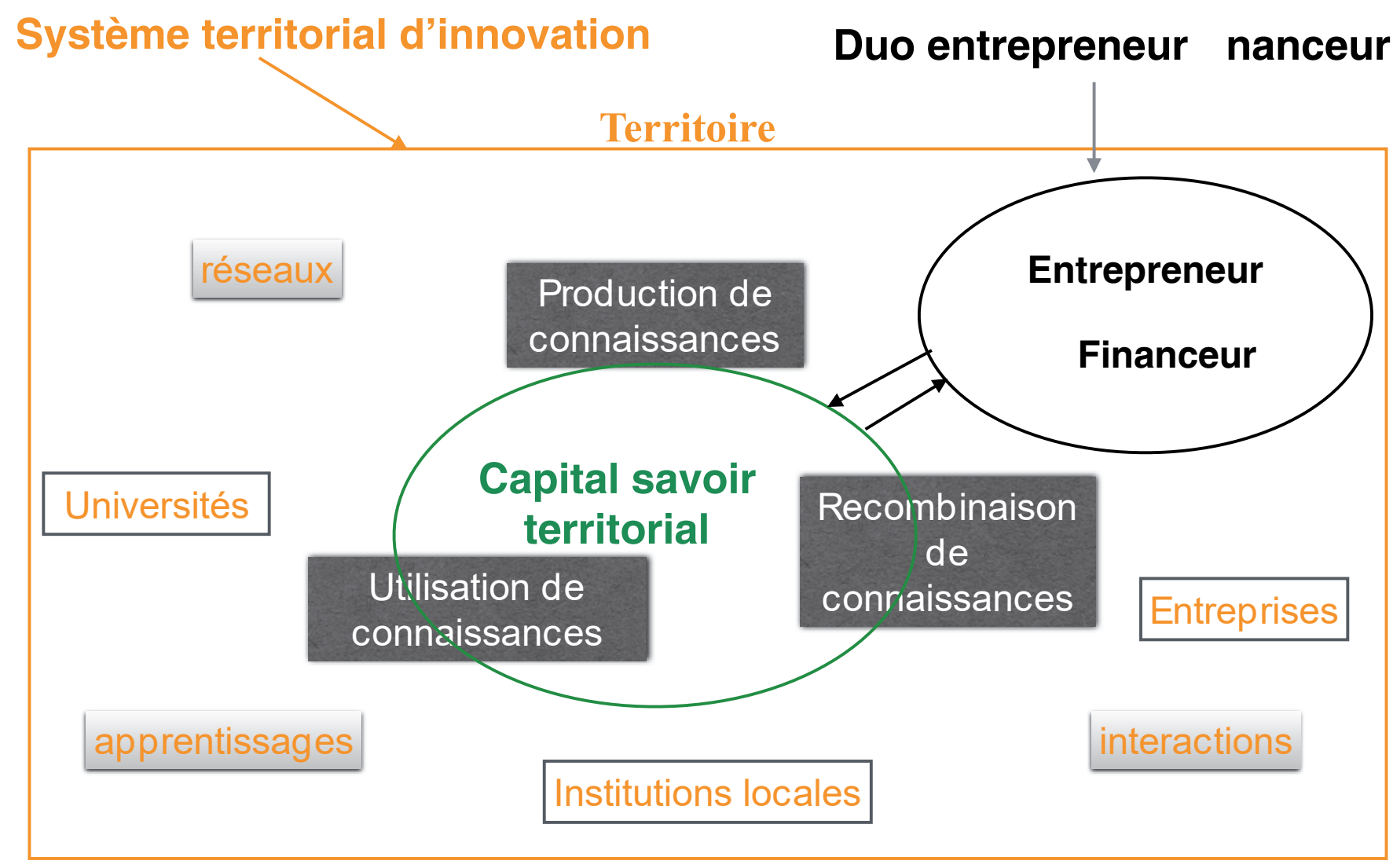

Figure 2. Les processus en œuvre au sein du système territorial d'innovation. Source : Auteur

Le système territorial d'innovation en stimulant les interactions, l'apprentissage, la mise en réseau permet la production, l'acquisition, la recombinaison et la systématisation des informations et connaissances scientifiques et techniques. 
Le duo entrepreneur investisseur par l'agrégation de ses connaissances et compétences au sein du système territorial d'innovation favorise l'augmentation du capital savoir territorial.

\section{Conclusion}

L'investisseur associé à l'entrepreneur est un catalyseur qui transforme la connaissance en entreprise et le capital risque augmente les chances de succès de la création d'entreprise innovante. Les relations sont multiples et s'inscrivent dans des logiques d'interactions, d'apprentissages et de collaborations plus que dans des logiques de contrat, ce qui permet plus d'agilité. La relation entrepreneur investisseur nécessite donc une analyse qui va au-delà d'une vision contractuelle. La caractéristique principale que nous retenons pour la construction de la définition du duo entrepreneur investisseur est la poursuite d'un but commun : le succès du projet d'entreprise dans un horizon de temps partagé. Le duo va dès lors mettre en commun des moyens pour parvenir à cet objectif. La mise en commun de moyens est favorisée par l'environnement dans lequel évoluent les deux acteurs à savoir le système territorial d'innovation. La relation entre entrepreneur et investisseur s'inscrit dans une dialectique avec le territoire.

Le système territorial d'innovation en stimulant les interactions, l'apprentissage, la mise en réseau permet la production, l'acquisition, la recombinaison et la systématisation des informations et connaissances scientifiques et techniques. Le duo entrepreneur investisseur au sein du système territorial d'innovation favorise l'enrichissement et la valorisation du capital savoir territorial.

Les relations entrepreneur investisseur peuvent résulter d'interactions et collaborations au sein d'un territoire, ce qui diffère de la vision habituellement présentée de la relation investisseur entrepreneur à savoir la théorie de l'agence et les problématiques d'asymétrie d'informations. L'analyse de la relation sous cet angle permet de montrer que de la relation entrepreneur investisseur peut permettre une adaptation aux changements et une agilité importante, nécessaire à la réussite du projet d'entreprise. Cette agilité se traduit par la capacité à adapter la relation en fonction des besoins de l'entreprise pour assurer son développement, par la production et la recombinaison de connaissance, et la recherche d'amélioration de la relation par les deux parties du duo. D'un point de vue théorique, cette communication propose une nouvelle approche de la relation entre entrepreneur et investisseur, en croisant les théories de la géographie économique évolutionniste et les approches de la «knowledge spillover entrepreneurship » que l'on définit comme le duo entrepreneur investisseur.

Les relations entrepreneur investisseur de types interaction et collaboration au sein d'un territoire permettent une plus forte adaptation aux changements et une agilité plus importante.

\section{Bibliographie}

[ACS 09] ACS Z.J., Audretsch, D.B., BRAUnERHJEL M., BRAUnERHJEL B., « The knowledge spillover theory of entrepreneurship », Small Business Economics, vol 32, pp 15-30, 2009.

[ACS 12] ACS Z.J., Audretsch D.B., BRAUNERHJEL M., «Growth and entrepreneurship », Small Business Economics, 39: $289,2012$.

[AKE 70] AKERLOF G., «The Market for Lemons: Quality Uncertainty and the Market Mechanism », Quarterly Journal of Economics 84 (3): 488-500, 1970.

[AUD 11] AUDRETSCH D.B., GRILO I., THURIK A.R., « Globalization, Entrepreneurship and the Region » dans Handbook of Research on Entrepreneurship and Regional Development, Cheltenham, Edward Elgar, M. Fritsch (ed.), 2011.

[AYD 85] AYDALOT, P., Economie Régionale et urbaine, Economica, Paris, 1985.

[BAD 97] BADOT O., Théorie de l'entreprise agile, L'Harmattan, 1997.

[BAR 11] BARZI R., « PME et agilité organisationnelle : étude exploratoire », Innovations, vol 35, pp 29-45, 2011 
[BER 98] Bergemann D., Hege, U., «Venture capital financing, moral hazard and learning ». Journal of Banking and Finance, 22(6-8), 703-735, 1998.

[BES 04] Bessant J., Francis D., Meredith S., KAPLinsKy R., Brown S., « Developing manufacturing agility in SMEs », International Journal of Technology Management, 22 (1-3), 28-54, 2004.

[BOU 17] BOUTILliER S., UzUNIDIS D., " L'équation entrepreneuriale dans l'économie contemporaine », dans Entrepreneuriat et Innovation Contextes et Concept, dir S. Boutillier, Peter Lang (ed), 2017.

[CAB 97] CABLE D.M., SHANE S., « 'A prisoner's dilemma approach to entrepreneur- venture capitalist relationships », Academy of Management Review, Vol. 22 (1), pp. 142-177, 1997.

[CAR 09] CARAYANNIS E.G., CAMPBELl D.F.J, "'Mode 3' and 'Quadruple Helix': toward a 21st century fractal innovation ecosystem», International Journal of Technology Management, Vol.46, n³, p.201-234, 2009.

[CAR 14] BoschMA R., «Competitiveness of regions from an evolutionary perspective », Regional Studies, vol 38, ${ }^{\circ}$, pp.1001-1014, 2004.

[CAR 14] CARLINO G., KeRR W.R., «Agglomeration and innovation ». Harvard Business School Working Paper no. 15$007,2014$.

[CHE 11] ChEMmANUR T.J., KRISHAN K. AND NANDY D.K., «How Does Venture Capital Financing Improve Efficiency in Private Firms? A Look Beneaththe Surface», The Review of Financial Studies, Vol. 24(12), pp. 4037-4090, 2011.

[COO 01] COOKE P., « Regional innovation system : comparative regulation in the new Europe », Geo Forum, vol 23, n ${ }^{\circ}$ 3, pp.365-392, 2001.

[COO 92] COOKE P., «Regional Innovation System, Clusters and Knowledge Economy », Industrial and corporate changes, vol 10, $\mathrm{n}^{\circ} 4$, pp 945-974, 1992

[COR 03] CORONADO A. E., "A framework to enhance manufacturing agility using information systems in SMEs », Industrial Management \& Data Systems, 103 (5), pages 310-323, 2003.

[DUB 06] DUBOCAGE E., « L'évaluation de la "start-up" par le capital-risqueur entre objectivité, jugement et mimétisme », Vie \& sciences de l'entreprise, 173, (4), 9-18, 2006.

[ELI 09] ELITZUR R., GAVIOUS A., "Contracting, signaling and moral hazard: a model of entrepre-neurs, angels and venture capitalists », Journal of business venturing, vol 18, pp 709-725, 2009.

[FLO 88] FLORIDA R., KENNEY M., «Venture capital and high technology entrepreneurship », Journal of Business Venturing, 3(4), pp.301-319, 1988.

[GOL 95] GOLDMAN S., NAGEL R., PREISS K., Agile competitors and virtual organizations: Strategies for enriching the customer, New York: Van Nostrand Reinhold, 1995.

[GOM 01] GOMPERS P., LERNER J., «The Venture Capital Revolution », Journal of Economic Perspectives, vol. 15-2, Spring, pp. 145-168, 2001.

[GOM 99] GOMPERS P., LERNER J., The Venture Capital Cycle, Cambridge: MIT Press, 1999.

[GRI 14] GRILLI L., MURTINU S., «Government, venture capital and the growth of European high-tech entrepreneurial firms ». Research Policy, 43(9), pp.1523-1543, 2014.

[GRO 93] Grossmann G.M., HelPMAn E., Innovation and growth in the global economy, MIT Press, Cambridge, 1993.

[HEG 98] Hege U., BergemanN D., «Venture capital financing, moral hazard, and learning ». Journal of Banking and Finance, Elsevier, vol.22, n6, pp. 703-735, 1998.

[HEL 00] HeLlmanN, T., PURI, M., «The interaction between product market and financing strategy: The role of venture capital». The Review of Financial Studies, 13(4), 959-984, 2000.

[HEL 02] HelmanN, T., PURI, M., «Venture capital and the professionalization of start-up firms: Empirical evidence». The journal of finance, vol. 57, no 1, p. 169-197, 2002.

[JEN 76] JENSEN M. C., MECKLING W. H., «Theory of the firm: Managerial behaviour, agency costs, and ownership structure », Journal of Financial Economics, 3(4), 305-360, 1976.

[KIN 93] KING R., LEVINE G., «Finance and growth: Schumpeter might be right ». Quarterly Journal of Economics 107, 717-737, 1993.

[KLA 05] KLAGGE B., MARTIN R., «Decentralized versus centralized financial systems : is there a case for local capital markets ?», Journal of Economic Geography, vol. 5, n 4, pp. 387-421, 2005. 
[KOR 00] KORTUM S., LERNER J., «Does Venture Capital Spur Innovation? », Rand Journal of Economics 31, 2000.

[LAP 07] LAPERCHE B., " 'Knowledge capital' and innovation in global corporations », International Journal of Technology and Globalisation (3)1, pp.24-41, 2007.

[LAP 16] LAPERCHE B., " Large Firms' Knowledge Capital and Innovation Networks », Journal of the Knowledge Economy, pp.1-18, 2016.

[LAP 17] LAPERChe B., Perrin-BoulonNe H., " Trajectoires, entrepreneurs et capital savoir territorial » dans Entrepreneuriat et Innovation Contextes et concept, dir S. Boutillier, pp 161-187, Peter Lang (ed), 2017.

[LEV 97] LEVINE R., «Financial development and economic growth: Views and agenda », Journal of Economic Literature 35, 688-726, 1997.

[MAC 87] MCMiLlian I.C., ZEMANN L., SUBBANARASIMHA P.N., «Criteria distinguishing successful from unsuccessful ventures in the venture screening process», Journal of Business Venturing, 2 (1987), pp. 123-137, 1987.

[MAN 13] MANigART, S. Wright, M., «Venture capital investors and portfolio firms». In Foundations and Trends in Entrepreneurship 9 (4-5). p.365-570, 2013

[MAS 02] MASON C., HARRISON R., « Is it worth it? The rates of return from informal venture capi-tal investments ». Journal of Business Venturing, 17(3), 211- 236, 2002.

[MAS 11] MASON C., HARRISON R., «Strategies for expanding Informal Venture Capital Market », International Small Business Journal, vo 11, $\mathrm{n}^{\circ}$ 4, 2011.

[MEI 14] MEIRRIEKS D., «Financial Development and Innovation: Is there Evidence of a Schumpeterian Finance Innovation Nexus? », Annals of Economics and Finance, 15-2, pp 343-36, 2014.

[NAN 15] Nanda R., Young K., Fleming L., «Innovation and entrepreneurship in renewable energy» dans In The Changing Frontier: Rethinking Science and Innovation Policy, eds. Jaffe, A., and Jones, B., National Bureau of Economic Research, 2015.

[PER 17] PERRIN-BOUlONNE H., « L'investisseur en capital de l'entreprise innovante dans les systèmes territoriaux d'innovation, les pôles de compétitivité comme terrain d'analyse », Cahiers du LabRII n³06, 2017.

[PUR 12] PURI, M., ZARUTSKIE, R., «On the Lifecycle Dynamics of Venture-Capital- and Non-Venture-Capital-Financed Firms», Journal of Finance, vol. 67, no. 6, pp. 2247-93, 2012.

[SAX 94] SAXENIAN A., Regional advantage: culture and competition in Silicon Valley and Route 128, Harvard University Press (ed), 1994.

[SCH 34] SCHUMPETER J., The Theory of Economic Development: An Inquiry into Profits, Capital, Credit, Interest and the Business Cycle. Cambridge, M.A.: Harvard University Press, 1934.

[SHA 02] SHANE, STUART, «Organizational endowments and the performance of university start-ups», Management Sciences, 48 (2002), pp. 154-170, 2002

[TEE 97] TEece D.J., PISANo, G., Shuen, A., « Dynamic Capabilities and Strategic management», Strategic Management Journal, 18 (7), pp. 509-533, 1997.

[WIR10] WIRTZ P. «Beyond Agency Theory: Value Creation and the Role of Cognition in the Rela-tionship Between Entrepreneurs and Venture Capitalists », dans Advances in Entrepreneurial Finance, Eds Springer, 2010. 